\title{
PARASITISMO DE GALLUS GALLUS (LINNAEUS, 1758) POR ESPÉCIES DE PHTHIRAPTERA EM CRIAÇÕES COLONIAIS NA REGIÃO SUL DO RIO GRANDE DO SUL, BRASIL
}

\section{L.S.S. dos Santos, L.M.J.F. Santos, C.L.G. Aguiar, J.L. Ruas, N.A.R. Farias}

Universidade Federal de Pelotas, Instituto de Biologia, Departamento de Microbiologia e Parasitologia, Laboratório de Parasitologia, Campus Universitário Capão do Leão, s/no, CEP 96010-900, Pelotas, RS, Brasil. E-mail: sssluciana@yahoo.com.br

\section{RESUMO}

Este estudo foi realizado com o objetivo de conhecer os piolhos (ordem Phthiraptera: Subordens Amblycera e Ischnocera) infestantes de galinhas de criações coloniais no Sul do Rio Grande do Sul. Cinquenta fêmeas adultas foram examinadas, de 10 propriedades rurais localizadas em 5 diferentes municípios. As aves foram eutanasiadas e posteriormente lavadas com água e detergente para coleta dos ectoparasitos através de filtragem por passagem em tamis com malha de $150 \mu \mathrm{m}$, separando-se em sedimento e sobrenadante, os quais foram preservados em etanol $70 \%$ até os processos de triagem e identificação. Cerca de 19.437 piolhos foram examinados e apresentaram a seguinte composição específica: os resultados indicam que a fauna de Phthiraptera em galinhas caipiras na região é composta por: Menopon gallinae (85,9\%), Goniodes dissimilis (6,1\%), Lipeurus caponis (3,0\%), Goniocotes gallinae (2,5\%), Menacanthus pallidus (2,1\%) e Menacanthus stramineus $(0,1 \%)$. Constatou-se que todas as aves examinadas estavam parasitadas por uma ou mais espécies de Phthiraptera, com predomínio de infestações múltiplas e moderadas (101 a 1.000 piolhos/ ave). M. gallinae é a espécie mais prevalente e abundante (100\% das aves parasitadas e média de 334,1 espécimes/ave). O grau de infestação das aves variou entre as propriedades, com médias de 41,2 a 680 piolhos/ave.

PALAVRAS-CHAVE: Piolhos mastigadores, Phthiraptera, galinhas caipiras, Estado do Rio Grande do Sul.

\begin{abstract}
PARASITISMOF GALLUS GALLUS (LINNAEUS, 1758) BY SPECIES OF PHTHIRAPTERA ON FREE-RANGE CHICKENS IN THE SOUTHERN REGION OF RIO GRANDE DO SUL, BRAZIL. This study was conducted in order to identify the species of biting lice (order Phthiraptera: Amblycera suborders and Ischnocera) that infest free-range chickens in southern Rio Grande do Sul, Brazil. Fifty adult females from 10 farms located in 5 different municipalities were examined for ectoparasites. The chickens were euthanized and then washed with water and detergent for the collection of ectoparasites by filtration using a $150-\mu \mathrm{m}$ mesh sieve, then separated into pellet and supernatant, which were preserved in ethanol $70 \%$ for the screening procedures and identification. A total of 19,437 lice were examined, presenting the following breakdown by species: Menopon gallinae (85.9\%), Goniodes dissimilis (6.1\%), Lipeurus caponis (3.0\%), Goniocotes gallinae (2.5\%), Menacanthus pallidus (2.1\%) and Menacanthhus stramineus $(0.1 \%)$. It was found that all the chickens examined were parasitized by one or more species of Phthiraptera, the multiple and moderate level of infestation (101 to 1,000 lice/chicken) being the most frequent. M. gallinae was the most frequent and abundant species (100\% of the chickens parasitized and an average of 334.1 lice/chicken). The degree of infestation of chickens varied among the farms, averaging 41.2 to 680 lice/chicken.
\end{abstract}

KEY WORDS: Biting lice, Phthiraptera, free-range chickens, Rio Grande do Sul State.

A avicultura em sistema extensivo (a campo) ou semi-intensivo (piquetes gramados) é uma das atividades agropecuárias mais efetivas para os agricultores familiares como fonte econômica e de proteína animal. Os alimentos gerados (carne eovos) são obtidos em curto espaço de tempo, requerendo baixos investimentos, com boa lucratividade e pouca ou nenhuma agressão ambiental, sendo essencial para suprir as necessidades proteicas do ser humano (GuERRA et al., 2008; Souza et al., 2009).

As galinhas domésticas são frequentemente infestadas por diversas espécies de ectoparasitos, especialmente piolhos (Insecta: Phthiraptera) (EMERson, 1956). A distribuição de cada espécie de piolho 
no corpo do seu hospedeiro ocorre em regiões ou nichos bem definidos (AsH, 1960).

Segundo PINTO et al. (2001), as aves infestadas tornam-se inquietas, não dormem bem e podem se autolesionar devido ao prurido provocado pelo caminhar dos parasitos, podendo gerar consideráveis impactos como a perda das penas, causando uma alteração térmica. Também podem ter sua alimentação afetada, ocasionando perda de peso e diminuição da capacidade reprodutiva, conforme GLESS; RAUN (1959) e Oliveira et al. (1999).

De acordo com VAlim et al. (2005), a ordem Phthiraptera se divide em quatro subordens, e entre elas apenas duas (Ischnocera e Amblycera) são encontradas nas aves. A grande diversidade de formas, com mais de 3.000 espécies conhecidas atualmente, é resultado de uma ampla variedade de hospedeiros, somada às adaptações necessárias para habitar diferentes nichos ecológicos.

As espécies das subordens Ischnocera e Amblycera são conhecidas comumente como piolhos mastigadores, alimentam-se de descamações cutâneas, plumas, secreções sebáceas e alguns gêneros de Amblycera ingerem sangue como complemento alimentar (CicChINO; CASTRO, 1998).

No Brasil, as principais espécies de piolhos encontradas em galinhas de criação colonial foram: Menopon gallinae (Linnaeus, 1758), Goniocotes gallinae (De Geer, 1778), Goniodes dissimilis (Denny, 1842), Colpocephalum turbinatum (Denny, 1842), Cuclotogaster heterographus (Nitzsch, 1866) e M. pallidus no RJ; (Neumann, 1912) (Oliverra etal.,1999), Menacanthhus stramineus (Nitzsch, 1818), Goniodes gigas (Taschenberg, 1879) e L. caponis (Linnaeus, 1758), com dominância de M. gallinae em SP (PINTO et al., 2001). GuerRA et al. (2008), na Ilha de São Luis, MA, identificaram: M. gallinae (31,33\%), M. stramineus $(2,48 \%)$, M. pallidus $(1,59 \%)$, Menacanthus cornutus (Schömmer, 1913) (0,18\%), L. caponis $(34,16 \%), G$. dissimilis (11,50\%), G. gallinae (17,70\%) e Menacanthus sp. $(1,06 \%)$.

CHIRINoset al. (2001), na Venezuela, identificaram nove espécies de piolhos, com as respectivas prevalências: $M$. stramineus (80\%), M. gallinae (51\%), L. caponis (43\%), Goniocotes gigas (Nitzsch, 1839) (32\%), G. gallinae (15\%), G. dissimilis (4\%), Colpocephalum sp. $(2 \%)$, G. gigas $(1 \%)$ e Chelopistes meleagridis (Linnaeus, 1758) (1\%). Os ectoparasitos Colpocephalum sp., Goniodes sp. e C. meleagridis foram descritos pela primeira vez no país.

FERRERO et al. (2004), na Argentina, detectaram M. stramineus (em $57 \%$ das aves examinadas), M. gallinae (em 43\%), L. caponis (em 43\%) e G. gallinae (em 29\%); os autores afirmaram que, conhecendo-se os tipos de ectoparasitos presentes nas aves, é possível recomendar medidas específicas de controle e de higiene no ambiente de criação para evitar a proliferação desses parasitos. MARÍN-Gómes; BENAVIDES-MONTAÑO (2007), na Colômbia, encontraram parasitismo por M. gallinae, G. gallinae, L. caponis, M. stramineus e G. dissimilis.

SYchra et al. (2008) examinaram 160 frangos de pequenas criações domésticas na região oriental da República Tcheca e calcularam as prevalências e intensidades de parasitismo de: G. gallinae (100\%; 110 piolhos), M. gallinae (88\%; 50), M. stramineus $(48 \% ; 17)$, L. caponis $(35 \% ; 12)$, M. cornutus $(12 \% ; 9)$, C. heterographus $(1 \%$; 4 ) e Goniocotes microthorax (Stephens, 1829) $(1 \%$; 3$)$. Evidenciaram que apenas duas aves de uma criação estavam fortemente infestadas pela espécie G. gallinae.

GONZÁLEZ-ACUÑA etal. (2009) confirmaram e atualizaram registros da presença dos Phthiraptera no Chile: M. gallinae, G. gigas, G. gallinae, M. stramineus e M. pallidus, sendo esta última espécie relatada pela primeira vez no país.

Tendo em vista a inexistência de estudos sobre a ectoparasitofauna de galinhas caipiras na região Sul do RioGrande doSul, o presente estudo foi realizado objetivando-se possíveis aplicações no manejo de criações coloniais.

Cinquenta galinhas domésticas (fêmeas adultas) obtidas em 10 propriedades rurais, duas em cada um dos seguintes municípios: Pelotas, Capão do Leão, Canguçu, Turuçu e Rio Grande, localizados entre os paralelos $-31^{\circ} \mathrm{e}-33^{\circ}$ (dados obtidos do IBGE) foram examinadas, considerando 5 galinhas por propriedade; todo material coletado de cada galináceo constituiu uma unidade de estudo.

Considerou-se pequena propriedade rural aquela em que o plantel não ultrapassava 80 aves, de linhagens variadas, onde as galinhas permaneciam soltas no campo durante o dia e a noite eram recolhidas em um abrigo para evitar o ataque de predadores e até mesmo o roubo.

Todas as aves foram adquiridas e eutanasiadas em um mesmo período. Após a morte, a separação da pele da carcaça foi efetuada através de esfolamento. A pele, juntamente às penas, foi submetida a um banho com uma solução de água e detergente líquido $(10 \mathrm{~mL} / \mathrm{L})$ e este material foi posteriormente peneirado, utilizando-se tamis com malha $150 \mu \mathrm{m}$. O material obtido através do banho foi separado em 100 frascos catalogados por unidade, sendo dois para cada galináceo, contendo sedimento e sobrenadante. Esses foram acondicionados em recipientes com uma solução de etanol $70 \%$ earmazenados no Laboratório de Parasitologia, Departamento de Microbiologia e Parasitologia do Instituto de Biologia da Universidade Federal de Pelotas.

Todo o conteúdo foi triado separando-se pequenas quantidades de sedimento e/ou sobrenadante que foram colocadas em uma placa de Petri. Em seguida, examinado por estereomicroscopia e efe- 
tuada a separação dos espécimes. Posteriormente, foram eles acondicionados separadamente por grupo taxonômico, em tubos de ensaio identificados por indivíduo hospedeiro. As identificações das espécies foram processadas com o auxílio de chaves de identificação (EMERSON, 1956; Price, 1977; TuFF, 1977). Alguns exemplares foram microfotografados e mensurados pelo programa Axio Vision 3.1.

Após as identificações das espécies, os parâmetros epidemiológicos de frequência, abundância e intensidade média de parasitismo foram calculados segundo Bush et al. (1997). Para designar os graus de infestação foram utilizadas as categorias sugeridas por De VANEY (1986): negativo - ausência de piolhos; leve - um a 100 piolhos; moderado - 101 a 1.000 piolhos; e severa - > 1.001 piolhos.

Seis diferentes espécies de Phthiraptera foram encontradas, com as respectivas frequências: $M$. gallinae (85,9\% dos exemplares identificados), $G$. dissimilis $(6,1 \%)$, L. caponis $(3,0 \%)$, G. gallinae $(2,5 \%)$, M. pallidus (2,1\%) e M. stramineus $(0,1 \%)$.

Os resultados concordam com os obtidos por GUERRA et al. (2008) que afirmaram que a fauna de Phthiraptera apresenta diversidade quanto a gêneros e espécies, dependendo das regiões geográficas analisadas. Segundo esses autores, as espécies mais frequentes na literatura são $M$. gallinae, $M$. stramineus, M. pallidus, L. caponis, G. dissimilis e G. gallinae, além de $M$. cornutus e seis outras espécies do gênero Menacanthus sp., cuja prevalência varia entre regiões fisiográficas de ocorrência.

Em galinhas de granja, OliveIRA; RIBEIRO (1990) verificaram que $M$. cornutus foi a espécie mais frequente no Estado do Rio Grande do Sul. Essa espécie não foi encontrada nas aves de criação colonial examinadas neste estudo, nem no realizado por OliverRa et al. (1999) no Rio de Janeiro.

Neste trabalho, todas as aves examinadas estavam parasitadas por uma ou mais espécies de Phthiraptera, sendo encontradas até 6 espécies parasitando a mesma ave. M. gallinae foi a mais prevalente e abundante $(100 \%$ das aves parasitadas e média de 334,1 espécimes/ave) e M. stramineus a menos frequente ( $12 \%$ - 0,44 espécimes / ave) (Tabela 1). Um total de 19.437 piolhos foi colecionado nas galinhas infestadas. Quanto ao grau de infestação, constatou-se que a maioria das aves $(70 \%)$ apresentava infestação moderada (101 a 1.000 piolhos/ave), $26 \%$ com infestação leve ( 1 a 100 piolhos/ave) e $4 \%$ (duas aves) com infestação severa, isto é, com mais de 1.000 exemplares de piolhos.

M. stramineus é uma das espécies mais patogênicas de Phthiraptera e geralmente apresenta-se em baixas infestações (SYCHRA et al., 2008), a exemplo do verificado nesse estudo (em $12 \%$ das aves, e média de 0,44 piolhos/ave).

Com o total de 16.705 exemplares entre ninfas e adultos, a espécie $M$. gallinae foi a mais frequente e abundante, corroborando com os resultados obtidos nos estados de Rio de Janeiro (Oliveira et al., 1999; PINTO et al., 2001), Paraíba (Borges et al., 2001) e Pernambuco (RochA et al., 2001).Segundo o último autor, a dominância dessa espécieé uma tendência mundial.

Atualmente, na Europa, os piolhos mastigadores ocorrem somente em criações domésticas de fundo de quintal, onde seu impacto econômico é pequeno. Por isso a maioria dos trabalhos sobre esses piolhos é realizada na África, Índia, Turquia e América doSul, onde esse tipo de criação tem importância econômica, com indicação de M. gallinae como espécie dominante (SYCHRA et al., 2008).

Constatou-se que $98 \%$ das aves estavam parasitadas por mais de uma espécie de piolhos, sendo registrado parasitismo simples somente em uma ave. A associação parasitária mais frequente (50\%) foi com cinco espécies: M. gallinae, L. caponis, G. dissimilis, G. gallinae e M. pallidus.

De acordo com EMERSOn (1956), G. gallus é a espécie de ave que apresenta parasitismo simultâneo por maior diversidade de espécies de Phthiraptera, confirmado agora com 49 aves parasitadas por mais de uma espécie de Phthiraptera, das 50 analisadas.

O número total de Phthiraptera coletados por propriedade variou de 206 (1,05\% do total de piolhos examinados) a 3.400 exemplares $(17,49 \%$ do total de examinados) o que indica uma infestação média de 41,2 a 680 piolhos/ave. Separadamente, por município, a infestação média por esses ectoparasitos é indicada na Figura 1. Isso evidencia variação quanto ao grau de infestação das aves entre as propriedades.

Tabela 1 - Parâmetros epidemiológicos de espécies de Phthiraptera em galinhas da zona rural da região Sul do Rio Grande do Sul.

\begin{tabular}{lccc}
\hline Espécies & $\begin{array}{c}\text { Prevalência } \\
\text { (\% aves parasitadas })\end{array}$ & $\begin{array}{c}\text { Abundância } \\
\left(\mathrm{n}^{\mathrm{o}} \text { espécimes/ave }\right)\end{array}$ & $\begin{array}{c}\text { Intensidade média } \\
\left(\mathrm{n}^{\text {o }} \text { espécimes/hospedeiros infestados }\right)\end{array}$ \\
\hline M. gallinae & $100 \%$ & 334,1 & 334,1 \\
L. caponis & $94 \%$ & 11,84 & 12,59 \\
G. dissimilis & $90 \%$ & 24,04 & 26,71 \\
G. gallinae & $76 \%$ & 9,84 & 12,94 \\
M. pallidus & $76 \%$ & 8,48 & 11,15 \\
M. stramineus & $12 \%$ & 0,44 & 3,6 \\
\hline
\end{tabular}




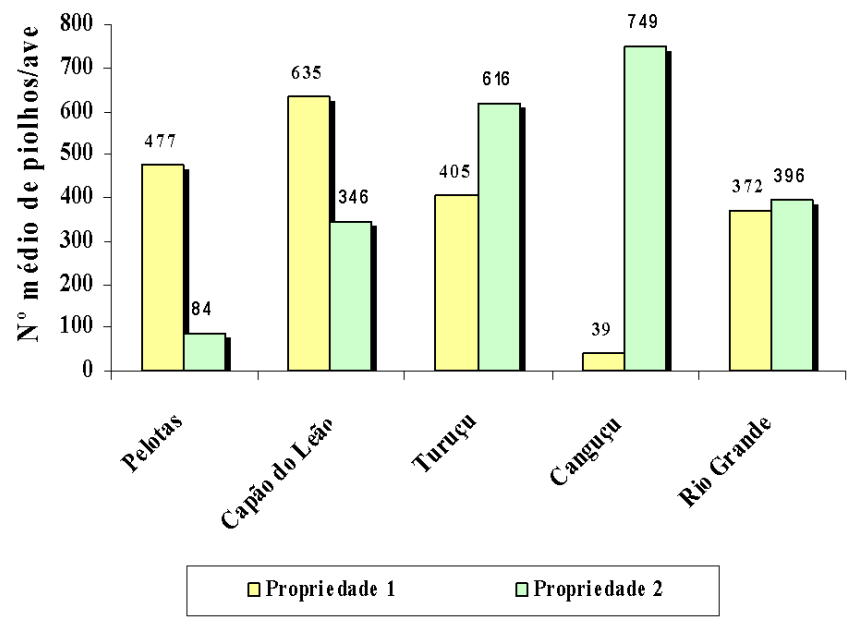

Fig. 1 - Infestação média por Phthiraptera em galinhas de criações coloniais de diferentes municípios do Sul do Rio Grande do Sul.

Independentemente da espécie considerada, observou-se maior frequência $(59,5 \%)$ de estágios imaturos (ninfas) em relação aos piolhos adultos. A relação macho/fêmea foi de 1:1,04. Resultados similares foram constatados por GUERRA et al. (2008) ao noticiarem $47,2 \%$ de ninfas em relação aos adultos e razão sexual de 1:1,6. Esses autores inspecionaram 40 aves, fizeram coleta de tufos de penas de diferentes regiões do corpo e obtiveram 565 exemplares entre ninfas e adultos. No presente estudo, o banho da pele com as penas, das 50 galinhas, proporcionou encontrar 19.437 espécimes, o que pode ser um indicativo de que esse método seja mais recomendável, capaz de capturar maior número de exemplares de Phthiraptera, e permitir avaliar melhor a intensidade de infestação nas populações de hospedeiros.

Os resultados obtidos permitem concluir que:

- todas as aves examinadas estavam parasitadas por Phthiraptera, com predomínio (70\%) de infestações moderadas;

- a diversidade de Phthiraptera é formada por: $M$. gallinae - a mais prevalente eabundante, G. dissimilis, L. caponis, G. gallinae, M. pallidus e M. stramineus, esta ocorre, dentre as espécies consideradas de maior patogenicidade, com baixas infestações;

- o parasitismo por múltiplas espécies de Phthiraptera ocorre em $98 \%$ dessas aves, sendo a associação com cinco espécies a mais frequente (50\% das aves), incluindo: M. gallinae, L. caponis, G. dissimilis, G. gallinae e M. pallidus;

- o grau de infestação das aves variou entre as propriedades, indicando a necessidade de medidas eficazes para o controle desses parasitos.

Este trabalho foi realizado conforme os princípios éticos da experimentação animal estabelecidos pela Comissão de Ética em Experimentação Animal da UFPel, sob o processo de número 23110.002475/2009-35

\section{REFERÊNCIAS}

ASH, J.S. A study of the Mallophaga of birds with particular reference to their ecology. Ibis, v.102, p.93-110, 1960. BORGES, S.S.; AMORIM, M.G.R.; FACCINI, J.L.H.

Estudo do parasitismo por malófagos (Insecta, Phthiraptera) em Gallus gallus na cidade de Patos, PB. Jornal Brasileiro de Patologia e Medicina Laboratorial, v.37, n.57, 2001.

BUSH, A.O.; LAFFERTY, K.D.; LOTZ, J.M.; SHOSTAK, A.W. Parasitology meets ecology on its own terms: Margolis et al. revisited. The Journal of Parasitology, v.83, n.4, p.575-583, 1997.

CHIRINOS, N.I.M.; CHIRINOS, A.R.; HINESTROZA, Y.; INICARTE, M.F.; MANCO, M.; MELÉNDEZ, A. Prevalencia de ectoparásitos en gallinas de corral (Gallus gallus domesticus) del municipio San Francisco, Estado Zulia, Venezuela. Revista Científica, FCV-LUZ, v.11, n.4, p.348-354, 2001.

CICCHINO, A.C.; CASTRO, D.C. Ischnocera. In: MORRONE, J.J.; COSCARÓN, S. (Ed.). Biodiversidad de artrópodos argentinos. Buenos Aires: Ediciones Sur, 1998. p.4-124.

DE VANEY, J. Ectoparasites. Poultry Science, v.65, p.649656, 1986.

EMERSON, K.C. Mallophaga (chewing lice) occurring on the domestic chicken. Stillwater, Oklahoma. Journal of the Kansas Entomological Society, v.29, n.2, p.63-79, 1956.

FERRERO, A.A.; GUTIÉRREZ, M.M.; GARCÍA, S.H.; CASTRO, D. Phthiraptera (Arthropoda, Insecta) en Gallus gallus (Galliformes, Phasianidae) en criaderos de áreas urbanas y suburbanas de la ciudad de Bahía Blanca, provincia de Buenos Aires, Argentina.

Entomología y Vectores, v.11, n.2, p.297-303, 2004. 
GLESS, E.E.; RAUN, E.S. Effects of chichen body louse infestion on egg production. Journal of Economic Entomology, v.52, n.2, p.358-359, 1959.

GONZÁLEZ-ACUÑA, D.; LARA, J.; CHICCHINO, A. Nuevos registros de piojos (Insecta: Phthiraptera) en aves domésticas y ornamentales en Chile. Archivos de Medicina Veterinaria, v.41, p.181-184, 2009.

GUERRA, R.M.S.N.C.; CHAVES, E.P.; PASSOS, T.M.G.; SANTOS, A.C.G. Espécies, sítios de localização, dinâmica e estrutura de populações de malófagos em galinhas caipiras (Gallus gallus L.) criadas na Ilha de São Luis, MA. Neotropical Entomology, v.37, n.3, p.259-264, 2008.

MARÍN-GÓMEZ, S.Y.; BENAVIDES-MONTAÑO, J.A. Parásitos en aves domésticas (Gallus domesticus) en el Noroccidente de Colombia. Arquivo Brasileiro de Medicina Veterinária e Zootecnia, v.1, n.2, p.43-51, 2007.

OLIVEIRA, C.M.B.; RIBEIRO, V.L.S. Ocorrência de Menacanthus cornutus (Mallophaga: Menoponidae) em galinhas do Rio Grande do Sul. Arquivo Brasileiro de Medicina Veterinária e Zootecnia, v.42, n.2, p.121-126, 1990.

OLIVEIRA, H.H.; FERREIRA, I.; SERRA-FREIRE, N.M. Fauna de Mallophaga (Insecta: Aptera) de ectoparasitos em Gallus gallus L. e Columba livia L. amostrados no Rio de Janeiro. Entomología y Vectores, v.6, n.5, p.509-515, 1999.

PINTO, C.; POSSATI, M.; VILLAÇA, A.; GUERIM, L.; SÁ-FREIRE, L.; SERRA-FREIRE, N.M. Ocorrência de malófagos em galinhas caipiras e sua relação com o padrão de coloração da plumagem. Entomología y Vectores, v.8, n.3, p.295-301, 2001.
PRICE, R.D. The Menacanthus (Mallophaga: Menoponidae) of the passeriformes (Aves). Journal of Medical Entomology, v.14, n.2, p.207-220, 1977.

ROCHA, P.M.C.; ROCHA JUNIOR, M.A.; TEIXEIRA, M.N.; MOTA, E.A.; OLIVEIRA, J.B.O. Perfil parasitológico de galinhas caipiras no Estado de Pernambuco. Jornal Brasileiro de Patologia e Medicina Laboratorial, v.37, p.227, 2001.

SANTOS-PREZOTO, H.H.; SILVA, M.O.; DAEMON, E.; D’AGOSTO, M.; PREZOTO, F. Sítios de localização de ectoparasitos em Gallus gallus Linnaeus, 1758. Revista Brasileira de Zoociências, v.5, n.1, p.129-135, 2003.

SOUZA, N.A.; OLIVEIRA, J.F.; HOLANDA, J.S.; CHAGAS, M.C.M.; FILHO, J.A. Ave caipira. Rio Grande do Norte: Empresa de Pesquisa Agropecuária do RN, 2009. 31p.

SYCHRA, O.; HARMAT, P.; LITERÁK, I. Chewing lice (Phthiraptera) on chickens (Gallus gallus) from small backyard flocks in the eastern part of the Czech Republic. Veterinary Parasitology, v.152, p.344-348, 2008.

TUFF, D.W. A key to lice of man and domestic animals. Texas Journal of Science, v.20, p.1-4, 1977.

VALIM, M.P.; TEIXEIRA, R.H.F.; AMORIM, M.; SERRAFREIRE, N.M. Malófagos (Phthiraptera) recolhidos de aves silvestres no Zoológico de São Paulo, SP, Brasil. Revista Brasileira de Entomologia, v.49, n.4, p.584-587, 2005.

Recebido em 26/10/11

Aceito em 12/4/13 